\title{
Physcial Characterization of Molybdenum microtips Field Emitter arrays
}

\author{
F. Cipriani
}

ESA/ESTEC, Keplerlaan 1, 2209 AG Noordwijk, The Netherlands

F. Leblanc,J.M. Illiano, J.J. Berthelier

LATMOS/IPSL, 4 avenue de Neptune, 94107 Saint-Maur-des Fossés, France

F. Müller

CEA/LETI, 17 rue des Martyrs, Grenoble, France

Résumé. We used a dedicated hemispherical energy analyzer to measure energetic and angular distributions of electrons emitted from Molybdenum microtips integrated in a $1 \mathrm{~cm}^{2}$ Field Emitter Array designed by the CEA/LETI laboratory. Such cathodes typically deliver about $25 \mathrm{~mA}$ at an extraction voltage of $100 \mathrm{~V}$, and are studied in order to replace heated wires as electron sources for space applications. We find that the energy distribution of the beam strongly depends on the extraction voltage, and is therefore expected to vary across the emission life time of the device, at a rate depending both on the alteration of the resistive structure with time, and on the fate of adsorbed contaminants at the tip surface. A semi-empirical model of the emitters is proposed and used to determine parameters of energetic and angular distributions. The energy dispersion of the beam is found to increase from $2 \mathrm{eV} \pm 20 \%$ up to $9 \mathrm{eV} \pm 20 \% \mathrm{eV}$, for extraction voltages varying from 40 to $100 \mathrm{~V}$. The mean angular dispersion of the beam is found to be $42^{\circ} \pm 20 \%$ when a null electric field is set at the grid extraction surface.

PACS numbers: 1315, 9440T

Submitted to: J. Phys. D: Appl. Phys. 


\section{Introduction}

Most of mass spectrometers on space missions have used electron impact ion sources based on thermoionic electron guns. Thermoionic sources provide electron current up to typically 500 $\mu A$, requiring several watts of power to heat the emitting filament up to typically $2000 \mathrm{~K}$. High power consumption, filament burning, and outgassing of heated materials in the source are the main shortcomings of the hot filament technique. Cold electron emitters such as Field Emitter Arrays (FEA) originally developed for flat screen applications, offer in that sense interesting perspectives for the next generation of space instruments.

In the present study we test Molybdenum microtips field emitters. Such cathodes have been extensively studied in the context of vacuum microelectronics applications such as flat panel display, microwave power tubes, or pressure gauges (Temple, 1999), and their performance continuously improved over the past decades (Schlesser, 1997). In particular several papers describing their current-voltage characteristics, emission area, emission stability as a function of exposition to different gases and pressures, can be referred to (Spindt et al., 1976; Brenac et al., 1987; Constancias, 1998).

Critical reliability issues have been pointed out (see for instance Aplin et al. (2004) for silicon field emitters) originating from various phenomena as degradation due to ion bombardment, arcing, or thermal breakdown due to electromigration. The present study 


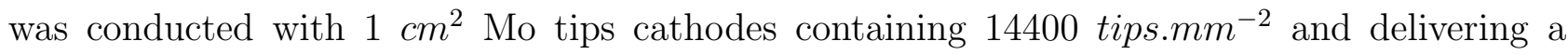
25mA nominal current. The samples were fabricated at CEA-LETI in Grenoble, France, using lithographic technique to obtain Spindt type tip emitters Constancias (1998).

Hot filaments have the advantage of emitting electrons with very low energies that can be accelerated and focused with a simple electrostatic optics. This is not the case of FEA emitters due to the field effect which causes electron extraction. In order to design ion sources it is thus necessary to have a detailed knowledge of the properties of the electron beam such as its energy or angular distribution. The knowledge of such parameters also allows to define optimized ion sources geometries and optics potentials in order to precisely control the electrons energy and spatial distribution of the electronic charge in the source. This is essentially done to obtain large ionization efficiencies, useful when probing low density media in space. For this purpose we set up an experiment based on an hemispheric energy analyzer, allowing a simultaneous measurement of the angular and energetic distributions of the emitted electrons (Cipriani, 2006). This experiment also allows to study the current stability as a function of the nature and pressure of the ambient atmosphere.

Previous field emission energy distribution measurements carried out by Brenac et al. (1987); Purcell et al. (1997), have revealed a complex behaviour due to processes affecting the structure of the material making the microtips as well as peculiarities in the design of the component. 
In the second and third sections of this paper we describe the emitter structure, as well as the experimental setup and principle of operation. In the fourth section we show energy and angular distributions measurements that are analyzed and modelled in the fifth section with the help of previous results from Baptist et al. (1997); Purcell et al. (1997). We actually show that due to the large width of energy distributions of emitted electrons, such cathodes are probably not the best candidates for an efficient design of ion sources.

\section{Description of the device}

The component is a square matrix composed of $32 \times 32$ independent pixels $\left(0.4 \times 0.4 \mu m^{2}\right)$ covering a total area of $11.2 \times 11.2 \mathrm{~mm}^{2}$. The extraction grids and tips (Figure 1) are polarized through two groups of Niobium tracks at right angle from each other. To ease the practical handling of the FEA, components have been glued into a Pin Grid Array (PGA) support using a UHV qualified cement. The Niobium tracks are bonded to the leads of the PGA. Molybdenum tips have an average height of $1 \mu m$ and an average half cone angle of $15^{\circ}$ (Constancias, 1998). The diameter of extraction grids apertures is $1.4 \mu m$ (cf. Figure 1). Extraction grids and cathodic contacts are isolated by a $1 \mu \mathrm{m}$ silica layer, as shown on Figure 3. A $800 \mathrm{~nm}$ silicon layer, referred to as the resistive sheet, is included in the structure between tips and cathodic contacts, in order to obtain good emission homogeneity, and to prevent destructive arcing between the tips and the extraction grids (Constancias, 1998). 
The electrical equivalent structure is shown on Figure 3. The equivalent resistance for the silicon layer between one tip and the cathodic contact through which it is biased has a value of $300 M \Omega$ (Muller, personal communication). This value has been shown to be current dependant, as a consequence of the variation of the equilibrium point of the voltages distribution across the tips (see Baptist et al. (1997) for more details), and also because of its semi-conductive nature. However its variations actually remain within $5 \%$ of the mean value, in the $0-100 \mathrm{~V}$ Vgc range. The complete electrical equivalent model shown on Figure 3 can be somehow simplified when considered in continuous voltage operation.

\section{Overview of the Experimental Setup}

The experimental setup is installed in a dedicated vacuum chamber with a base vacuum level of $10^{-8}$ mbar, achieved by turbomolecular pumping. Continuous measurements of the residual gas were carried out using a quadrupole mass spectrometer. Cathode baking was not performed before operation as we reproduced operations onboard a satellite where the large power necessary for heating would not be available. A satisfactory vacuum level of $2.3 \times 10^{-8}$ torr was obtained after two weeks of pumping. The vacuum composition is basically a mixture of the residual nitrogen used as the venting gas (41\%), and residual air. The potential interference of those gases on our measurements is very weak at this pressure level. We noted that degassing of $\mathrm{H}_{2}$ from the vacuum chamber walls remained fairly weak compared with the nitrogen level 
(about $25 \%$ of the nitrogen peak).

As shown on Figure 4 the main components of the experimental setup are: (1) a sample holder attached to a XY linear travel, (2) the Field Emitter Array, and (3) the hemispheric energy analyzer. Electrical connections were realized with Kapton insulated cables or with bare rods connected to feedthroughs on the chamber flanges. The PGA support is mounted on an alumina support followed by bare rods. The whole assembly can be moved in front of the analyzer entry plane along two X,Y perpendicular directions with a positioning resolution of $5 \mu m$.

Measurements of electrons characteristics were preformed with the retarding potential hemispheric analyzer, discussed in details in Cipriani (2006). In this setup (see Figure 4) the FEA is positioned in a plane parallel to the entrance plane of the analyzer, which has an entry hole $250 \mu \mathrm{m}$ in diameter. The distance between the cathode' surface and the entrance plane is $5 \mathrm{~mm}$. More than $99 \%$ of the emitted beam is collected on the entrance plane and the electrons passing through the entry hole enter the analyzer cavity within a $2^{\circ}$ aperture cone.

The inner surface of the analyzer entrance plate is insulating and supports 18 materialized equipotentials under the form of rings of width $1 \mathrm{~mm}$, polarized in such a way that the retarding field in the analyzer cavity is radial. Electrons entering the hemisphere are therefore decelerated by the radial retarding field along their trajectories. A potential energy diagram of the experiment is shown on Figure 2. The retarding field amplitude is set by the difference 
between the collector potential $V$ coll, and the potential $V d$ of the entrance plate of the analyzer. Electrons are emitted from an energy level theoriticaly close to the Fermi level of the tips, which are biased to potential Vp, and are accelerated towards the extraction grids, which are biased to a more positive potential $\mathrm{Vg}$. Therefore electrons energies reach a maximum value close to $\mathrm{e}(\mathrm{Vg}-\mathrm{Vp})-\Phi_{\text {grid }}$ at the grids surface (where $\Phi_{\text {grid }}$ is the work function of the grid). The collector is in stainless steel with a work function $\Phi_{\text {coll }}$ of $4.85 \mathrm{eV}$ according to Benenson et al. (2002). We estimate this value as representative of the actual work function of the collector within $\pm 0.5 \mathrm{eV}$. The collector's potential has to be varied from a value $V$ coll $_{\max }$ such that $V$ coll $_{\text {max }}>e(V g-V p)+\Phi_{\text {coll }}$, downto a value $V$ coll $_{\text {min }}$ such that $V \operatorname{coll}_{\min }<e(V g-V p)+\Phi_{\text {coll }}$. The cut-off condition corresponds to equality. The collector's potential is varied in $100 \mathrm{meV}$ steps, resulting in a non zero electron current when the retarding potential $V_{\min }$ is lower that the lowest energy of the electrons, and providing measurement of the energy distribution when varied from $V_{\min }$ to $V_{\max }$ (retarding potential preventing electrons to hit the collector). Moving the FEA in front of the entrance plane provides a at the same time measurement of the electron intensity as a function of the angle between the FEA tips axis and the central axis of the hemispherical analyzer. 


\section{Measurements}

\subsection{Measurement sequence}

We randomly selected a set of 5 pixels (out of 1024) on which measurements of the energetic and angular dispersions of the beam have been carried out. The coordinates of such pixels on the 32 columns (c) by 32 lines (l) matrix are: (c9,114), (c9,115), (c9,116), (c17,115), and (c27,18).

During our measurements cathodic contacts were biased at 0V, extraction grids were biased at a potential $\mathrm{Vg}$ varying between $+20 \mathrm{~V}$ and $+100 \mathrm{~V}$. The entrance plate was biased to a potential $\mathrm{Vd}$ equal to $\mathrm{Vg}+20 \mathrm{~V}$, such that the electric field is directed from the entrance plate towards the FEA, that is opposite to the retarding field in the analyzer. This allows to reduce the potential bubble forming near the entry hole and disturbing electron trajectories. Such biasing also prevent most of the secondary electrons emitted by the entrance plate to be lost by back scattering, ensuring a minimum error on the emitted current measurement. The potential of the protecting electrode (surrounding the emitting surface, in red in Figure 4) was set near 0V to produce a slight beam focusing effect and avoid hazardous electronic trajectories outside the analyzer volume. 


\subsection{Current voltage characteristic and emission stability}

In order to avoid destructive arcing, extraction grids potentials were operated with a duty cycle of $10 \%$. That is, the extraction voltage Vgc is applied during $1 \mathrm{~ms}$, every $10 \mathrm{~ms}$. The FEA is operated in a residual vacuum $\left(P \simeq 10^{-8}\right.$ mbar). After stablization of the Fowler-Nordheim characteristic, the emitted current density is $25 \mathrm{~mA} \cdot \mathrm{cm}^{-2}$ when the extraction voltage is $100 \mathrm{~V}$.

Figure 5 shows the typical evolution of the emitted current over a period of $30 \mathrm{~h}$ for a subset of 32 pixels forming one column of the emitter. The average current is $65.9 \mu \mathrm{A}$ with variations less than $2 \%$ during the measurement. A stabilized current-voltage characteristic of this column is plotted on Figure 6, the emitted current reaching $80 \mu A$ at $100 \mathrm{~V}$. The inset of Figure 6 shows a Fowler-Nordheim fit to the measured current. As can be observed, a slight deviation from the Fowler-Nordheim fit occurs at high voltage. Such deviation is typical for this type of emitter and results from the presence of a resistive sheet between cathodic contacts and tips. At high current values flowing through the resisitve layer, the resulting voltage drop at the tips lowers the emitted current compared with the Fowler-Nordheim current for the same voltage without resistive sheet. As will be shown in section 5, such resistive layer has direct consequences on the width of the energy distribution of emitted electrons. 


\subsection{Results of the energy analysis}

Figure 7 shows the evolution of the Collector current as a function of the collector voltage for pixel $(\mathrm{c} 17,115)$. The extraction voltage is successively set to 40, 60, 80 and 100V.

We observe that:

- the threshold voltage for current collection increases with the extraction voltage,

- the energy spread increases with the extraction voltage,

- mostly visible on the $100 \mathrm{~V}$ curve, appears a slight stepping behaviour of the collected current, consistent with a multi peaked energy spectra. Inter-peaks regions in the distribution indeed result in a local decrease of the current variation $\mathrm{dI} / \mathrm{dV}$, when the retarding field is varied.

As will be shown in detail in section 5 our observations are consistent with the Total Energy Distribution measurements by Purcell et al. (1997) :

- a shift is observed between the effective collection threshold voltage and the threshold expected if emitted electrons had energies close to eVgc. This shift increases with Vgc.

- the energy spread of the distribution increases with Vgc, reaching roughly $10 \mathrm{eV}$ at $100 \mathrm{~V}$ in our case.

A comparison of collected currents for the set of 5 pixels tested when biased at $60 \mathrm{~V}$ is shown in Figure 8. Though the amplitude of the total collected current varies by a factor of 7 , the 
variability over pixels of the mean values of the energy distributions deduced from the curves is not larger than $10 \%$ of the average mean energy value. Such variations are representative of variations observed at any extraction voltage between $40 \mathrm{~V}$ to $100 \mathrm{~V}$.

The factor of 7 in the current level at the collector reflects a variability of the total emitted current between pixels. Such variability can be related to a number of factors: structural discrepancies in the resistive material, the presence of surface contaminants, pixel individual history, variations of the number of active tips from one pixel to another. The relative importance of each one of those factors is however difficult to single out.

We monitored the temporal evolution of the energy spectra, for electrons emitted by pixels $(17,15)$ (green and black curves) and $(9,14)$ (red and yellow curves) over time intervals of respectively one month and 3 months. The corresponding effective emission times (involving operations at different extraction voltages and a constant pressure level) were respectively of 146 and 104 hours. Between the beginning and the end of those periods we observed a decrease of the mean energies of the distributions close to $2 \mathrm{eV}$ in the case of pixel $(17,15)$, and close to $1 \mathrm{eV}$ in the case of pixel $(9,14)$. This decrease is consistent with the alteration of the resistance between tips and cathodic contacts. Those measurements give indications on variations of the energetic structure of emitted electrons which are important to consider in order to optimize parameters of ion sources optics. 


\subsection{Results of the angular analysis}

Measurements presented here have been carried out at an extraction voltage of $60 \mathrm{~V}$. Electrodes potentials were set in the same configuration than described in section 4.2.

By simultaneously measuring the position of the sample holder central axis along two orthogonal X and Y axis (cf. Figure 4), and the collected current, we could derive the shape of spot due to the emitted beam on the analyzer entrance plane, as well as the angular dispersion of the beam. For that purpose the position of the emitting area with respect to the sample holder central axis has been measured and is known with a precision of $\pm 100 \mu m$. A numerical model of the experiment and emitters (see part 5) then allows to determine a beam angular profile.

Positioning the component from one step to the following takes on average 10 seconds. Since the temporal variability of the beam can be important with respect to this time scale, our observations are based on averaged current values at each $(\mathrm{X}, \mathrm{Y})$ position of the emitter.

Figure 9 shows a 3D profile of the beam (intensity as a function of the spatial coordinates on the entrance place) emitted by pixel $(9,15)$. The maximum amplitude of the average collected current measured in the pulsed mode is close to 500pA. The collected current reaches a few pA on the external boundary of the beam. We define the beam boundary by setting a threshold at $5 \%$ of the maximum current value. Figures 10 and 11 show normalized current profiles measured respectively along the $\mathrm{X}$ and $\mathrm{Y}$ axis, for the set of five pixels considered in this study. Each 
curve is an average profile of 3 passes of the emitter along the axis (each point is an average over five values successively acquired at 1 s time intervals). The distance travelled by the component between measurement positions is $1 \mathrm{~mm}$, in order to minimize the total integration time and variations of the emitted current over the whole sequence. As illustrated with pixel $(17,15)$ profile on Figures 10 and 11, increasing the spatial frequency to $0.5 \mathrm{~mm}$ does not significantly affect the profiles shape.

Normalized profiles shown on Figures 10 and 11 exhibit similar slopes, while maxima are shifted along the displacement axis, consistently following the emitting pixel position. Since electron trajectories depend on non homogeneous grids potentials and potentials of nearby electrodes, direct calculation of the angular profile is not possible from geometrical considerations only. Therefore we have used a semi-empirical model of the emitters, presented in the following section, which has been integrated into a numerical model of the experiment. Along the $\mathrm{Y}$ axis, the collected current decreases very steeply when the distance from the maximum position increases, with an average $2.25 \mathrm{~mm}$ width at half maximum and $4.61 \mathrm{~mm}$ on the entry plane of the analyzer. Along the $\mathrm{X}$ axis, the slope is shallower with an average 3.44 $\mathrm{mm}$ width at half maximum and $7.95 \mathrm{~mm}$ on the entry plane. This indicates an asymetrical repartition of the current with respect to the analyzer axis. Those values vary by about $20 \%$ over the set of tested pixels.

Clearly a homogeneous beam density can not be expected from those kind of emitters, 
as demonstated by beam section imaging on phosphorous targets in Purcell et al. (1997) and Constancias (1998). Indeed, the nanograin structure of emitting centres covering the tip surface as well as charging effects near the extraction grids affect the spatial sidtribution of the emitted current. However, those effects are smoothed out as the emitter number is increased, and can not be responsible for the observed asymmetry of the beam.

\section{Interpretation}

This section provides an interpretation of the data shown on Figure 7, 10 and 11. An emphasis is put on deriving parameters useful to optimize ion sources optics, such as those allowing to model energetic and angular distributions of emitted electrons.

\subsection{Energy distribution of emitted electrons}

As reported in Baptist et al. (1997) the resistive sheet (silicon layer shown on Figure 3) plays the role of an electrical ballast able to:(1) regulate emitted currents as a function of tips locations on the sheet, (2) allow a uniform emission, (3) protect the FEA from destruction through arcing between tips and the extraction grid. The resistive sheet also acts as a coupling device between tips, since current tubes flowing from each tip to the closest cathodic contact cross the common resistive structure. Such current induces a voltage drop between the emitting tip and the extraction grid, therefore affecting the potential of every other tip. Since one tip 
typically emits $1 \mu \mathrm{A}$ at an extraction voltage of $100 \mathrm{~V}$, with a resistance value of $10^{7} \Omega$ the voltage drop per tip reaches values higher than $10 \mathrm{~V}$.

Another study (Purcell et al., 1997) reports detailed measurements of the energetic structure of the emitted beam from 1 to $4 \mathrm{~mm}^{2} \mathrm{FEA}$, using a $135^{\circ}$ hemispherical energy analyzer with a nominal resolution of $10 \mathrm{meV}$, at four stages of operation of the FEA:(1) during the first emission, (2) during the seasonning process, (3) after the seasonning process, (4) before and after flash experiments. The base vacuum was $5 \cdot 10^{-11}$ torr and the samples were not baked before field emission, which is similar to our case. The observed energy spectra proved to be rapidly evolving with main and secondary peaks appearing and disappearing during the seasonning process. Increase of the extraction voltage caused an increase of the distance between peaks with typical widths of the whole distribution ranging from $10 \mathrm{~V}$ up to $30 \mathrm{~V}$.

Such observations were interpreted with a model accounting for the presence of nanometric structures on the surface of the tip material (relevant when tips surfaces are not cleaned by heating), and mobile surface contaminants. Following this model electrons are emitted from localized energy bands associated with those structures, which are energy-shifted with respect to the Fermi level of the tip material and between each other.

Emission features presented in this paper are consistent with observations reported both in Purcell et al. (1997) and in Baptist et al. (1997). Indeed, the mean energy of the beam inferred from Figure 7 is much lower than the value expected from clean tips with no resistive structure, 
and the energy spread is on the contrary much larger than the theoretical behaviour observed from single tips, reaching roughly $10 \mathrm{eV}$ at $100 \mathrm{~V}$ in our case.

5.1.1. Mean Energy of emitted electrons: Using a Gaussian distribution of the energy of emitted electrons in our simulation, we fitted the current curves shown on Figure 7. From those fits we obtain a range of energy dispersions of the beam between $1 \mathrm{eV}$ and $4 \mathrm{eV}$, and a range of voltage drops between 5 and $25 \mathrm{eV}$, when the extraction voltage varies between $40 \mathrm{~V}$ and $100 \mathrm{~V}$. Since our samples are not baked and very probably contaminated (our base vacuum level is larger by 3 orders of magnitude compared to that of Purcell et al. (1997)), effects of granular surface structures and contaminants certainly account for a significant part of the observed energetic spread.

On the tip scale, the resistive structure significantly influences the energy of emitted electrons. This structure is modelled on Figure 12, in which $R 1$ represents the resistance of the silicon layer between the cathodic contact and the tip base. Since the structure is symmetrical with respect to the tip axis, Figure 12 represents half of the electrode structure encompassing each tip. However resistance values indicated on this Figure refer to the complete structure. Currents are noted as following: $i_{e}$ is the total emitted current, $i_{g}$ is the current intercepted by extraction grids, $i_{f}$ is the current flowing from the cathodic contact towards the silica, which divides into currents $i_{f 1}$ and $i_{f 2}$. The current flowing through cathodic contact is noted 
$i_{c}$, and is the sum of $i_{e}$ and $i_{f} . R 2+\lambda R 1$ is the sum of the silica resistance $R 2$, and of an unknown resistance $\lambda R 1$ encountered in the resistive sheet by the leakage current flowing from the extraction grid to the cathodic contact $\left(i_{f 2}\right.$ in the silica layer and $i_{f}$ in the resistive sheet). It represents the isolation between the cathodic contact and the extraction grid. The resistance between the extraction grid and the tip is the sum of $R 2$ and of a lateral resistance, noted $\mu R 1$, corresponding to the physical volume of the resistive sheet contained in the current tube flowing from the insulator base to the tip base (corresponds to $i_{f 1}$ ). Measurements that we have carried out (see below) tend to show that a non negligible leakage current between the extraction grid and the cathodic contact is present during the emission process. The equilibrium voltages of the tips will therefore also depend on the behaviour of the resistive sheet regarding the leakage current. The potential at the silica/silicon interface associated with this current may therefore be different from the nearby tip potential, hence inducing an equilibrium current $i_{f 1}$.

We adopt here a simple resistive model to describe those different effects, assuming that resistances associated with currents $i_{f}$ and $i_{f 1}$ (which cross the resistive sheet as does $i_{e}$ ) can be seen as proportional to the resistance $R 1$.

We moreover consider that extraction grids collect very few electrons (the seasonning process of the tips is achieved). Therefore we assume a leakage current $i_{g}$ arising partly from such electrons, and from surface currents flowing on the silica and silicon exposed to the vacuum (due for instance to carbon deposition during the emission or fabrication process (Muller, personnal 
communication)), being less than $10 \%$ of the emitted current. This is consistent with current values observed from the difference between the collected current at the entrance plane of the analyzer, and the emitted current measured at the cathodic contact.

In order to estimate $R 2+\lambda R 1$, we carried out measurements of the pixel isolation when the cathode is not active. For those measurements the emitter is polarized in such a way that the field emission process is nullified (therefore $i_{f}=i_{f 2}$ ).

Assuming that the isolation resistance remains equal when the emitter is polarized in normal mode, we consider this measurement as a good estimate of $R 2+\lambda R 1$. Results for a set of 10 pixels are shown on Figure 13. The pixel isolation is observed to vary as a function of the extraction voltage. This is due to both the semi-conductive nature of the silica/silicon material, and to variations of the resistive sheet equivalent resistance with the extraction voltage as reported in Baptist et al. (1997). The range of observed values extends from several tens of $M \Omega$ near $0 \mathrm{~V}$ to about $2 M \Omega$ for $V g c=100 \mathrm{~V}$. This implies that a leakage current as high as $50 \mu \mathrm{A}$ per pixel is likely to occur in the cathodic contact at this voltage level.

We find that this behaviour can be modelled by a power law of the form:

$$
R 2+\lambda R 1=R_{0} \cdot V g c^{-\alpha}
$$

where $\alpha$ takes values between 0.4 and 0.5 , depending on the quality of the isolation at the moment of the measurement. Therefore such a leakage current crossing a resistive sheet of average resistance $170 k \Omega$ (estimate of the equivalent resistance for the whole pixel structure), 
leads to an average $8.5 \mathrm{~V}$ voltage drop at the tips, which is of the same order than the voltage drop due to the emitted current crossing the resistive sheet.

Indeed, in order to estimate an equivalent resistance for the pixel as seen by the emitted current we also have to consider that, due to the geometry involved, the current tube flowing from the tip base to the cathodic contact only affects the nearby area of the resistive sheet. Moreover, although there are 1760 tips per pixel, the proportion of emitting tips is actually close to $50 \%$ (Constancias, 1998). Therefore using a value for $R 1$ which is $300 M \Omega$ (Muller, personal communication), the equivalent resistance seen by the emitted current is $340 k \Omega$. This value causes again a mean $8.5 \mathrm{~V}$ voltage drop assuming an average emitted current of $25 \mu \mathrm{A}$ per pixel at an extraction voltage of $100 \mathrm{~V}$.

We can now estimate the mean voltage drop at the tips, writing the following expression of $\mathrm{Vg}-\mathrm{Vp}$ :

$$
V g p_{\text {modelled }}=V g-V p=(V g-V c)\left(1-\frac{1}{A}\right)-k \times i_{e} \frac{B}{A}
$$

where:

$$
A=\left[1+\mu+\left(1+\frac{1+\mu}{\lambda}\right) \frac{R 2}{R 1}\right] \quad B=\left[\mu R 1+R 2\left(1+\frac{\mu}{\lambda}\right)\right]
$$

Coefficient $\mathrm{k}$ accounts for the proportion of the emitted current collected by the extraction grids. In equation (2) $i_{e}$ represents the current measured at the analyzer entrance, we therefore assume that a current $k \times i_{e}$ (with $k>1$ ) is actually emitted. This has no effect on the 
model considered on Figure 12, but helps to figure out the influence of the intercepted current $\left(i_{g}=(k-1) i_{e}\right)$ on the potential difference $(\mathrm{Vg}-\mathrm{Vp})$.

Using measured values of $R 2+\lambda R 1$ for pixel $(17,15)$, we studied the influence of parameters $k, \lambda, \mu$, and $R 1$, on the potential difference $(\mathrm{Vg}-\mathrm{Vp})$. Results are summarized on Figure 14 .

On this figure the green line with squares shows the potential difference (Vg-Vp) associated with mean energy values $\mathrm{e}(\mathrm{Vg}-\mathrm{Vp})=\mathrm{e}\left(\mathrm{Vg}-\mathrm{Vc}_{\mathrm{c}}\right) \mathrm{e}\left(\mathrm{Vp}-\mathrm{Vc}_{\mathrm{c}}\right)$ of the beam deduced from data corresponding to pixel $(17,15)$ as shown on Figure 12 . We carried out a parametric study by varying $\mu$ from 0 up to $0.5, \lambda$ from 0.5 up to $2, \mathrm{R} 1$ from $170 k \Omega$ up to $680 k \Omega$ (equivalent to a proportion of emitting tips from $100 \%$ down to $25 \%$ ), and $k$ from 1.1 to 1.5 (around $10 \%$ of the emitted current in usually lost in the extraction grids during nominal operation of the FEA). On Figure 12 we plotted four sets of curves in the parameter space, illustrating the most important features. For each set of curves $\lambda$ has been given 4 values which are 0.5, 1, 1.5, and 2 . Increasing $\lambda$ reduces the discrepancy between the modelled and observed $(\mathrm{Vg}-\mathrm{Vp})$. Comparing the black set of curves (corresponding to $R 1$ value of $170 k \Omega$ ), with the cyan $(R 1=340 k \Omega)$, and the yellow $(R 1=680 \mathrm{k} \Omega)$ sets of curves, we note that increasing the proportion of emitting centres (all other parameters staying equal) increases the discrepancy between observed and modelled values of $(\mathrm{Vg}-\mathrm{Vp})$. Also, increasing $\mu$ from 0 to 0.2 increases this discrepancy, as can be seen from the red and blue sets of curves (the effect however remains smaller in this case). At last, increasing the leakage current at the grids from $10 \%$ to $50 \%$ of the emitted current 
decreases the discrepancy between the modelled and measured values, as can be observed from the cyan and blue sets of curves.

The main features derived from the modeled values of $(\mathrm{Vg}-\mathrm{Vp}$ ) (all of which are not shown here) are the following:

- the Vgp model usually overestimates the observed values: therefore the resistive structure alone can not explain the observed energy shift,

- a proportion of emitters lower than or equal to $50 \%$ is required to allow parameters $\lambda$ and $\mu$ to stay in a realistic range of values (that is $\lambda$ values close to 1 and $\mu$ values close to 0 ), - increasing the current intercepted by the grids lowers the discrepancy between model and observations.

We conclude that our model gives a satisfactory qualitative description of the resistive contribution to the mean energy of the emitted beam. Based on that, in order to build a phenomenological model of the emission, we now propose a simple statistical description that accounts for the effect of nanometric protrusions at the tip surface.

The potential difference $V p c$ between the tips and the cathodic contacts can be written as the sum of a resistive contribution $V p_{R}$ and a contribution due to the material granularity $V p_{n}:$

$$
V p c=V p_{R}+V p_{n}
$$


The $V p_{R}$ component describes the influence of both the resistive sheet and that of the insulating layer on the mean tip potential. From previous considerations we can write:

$$
V p_{R}=V g c_{\text {applied }}-V g p_{\text {modelled }}
$$

where $V g c_{\text {applied }}=V g-V c$ and $V g p_{\text {modelled }}$ in given by equation (2).

Equation (4) is constrained by the mean energies of the beam fitting the measurements shown on Figure 7. Those mean energy values are respectively $35.03 \mathrm{eV}$ (for $\mathrm{Vgc}=40 \mathrm{~V}$ ), $50.70 \mathrm{eV}$ (for $\mathrm{Vgc}=60 \mathrm{~V}), 64.20 \mathrm{eV}$ (for $\mathrm{Vgc}=80 \mathrm{~V}$ ), and $75.40 \mathrm{eV}$ (for $\mathrm{Vgc}=100 \mathrm{~V}$ ), and correspond to mean voltage drops at the tips shown on Figure 15. On that figure yellow triangles represent the observed values of the mean energy, and are adjusted by a second order polynomial plotted on the graph. The curve with squares shows corresponding Vgp values, obtained by subtracting the polynomial values from Vgc. The green curve with disks shows values of $V g p_{\text {modelled }}$, obtained with realistic values of our parameters such that $k=1.1, \mu=0.1, \lambda=1.0$, and $R 1=340 k \Omega$. Adjusted values of $\mathrm{Vpc}$ and $V p_{n}$ are respectively represented by the blue curve with diamonds, and by the red curve with stars.

According to this approach we obtain that nanometric surface structures accounts for about $50 \%$ of the mean value of $V p c$.

5.1.2. Energy dispersion of emitted electrons: While we previously worked on the pixel scale, we now scale the study on the tip scale in order to consider dispersion of the parameters inside 
one pixel. The resistive model proposed on Figure 12 is also valid on the tip scale.

Purcell et al. (1997) demonstrated that nanometric protrusions were responsible for a discrete and shifted peaking of the energy spectra therefore leading to a large overall energetic dispersion. We choose to describe such behaviour in a practical and representative way by using two parameters, the mean energy $e V p_{n}$ and the dispersion $\sigma_{n}$, associated to a gaussian envelope of the spectra noted $\Gamma\left(e V p_{n}, \sigma_{n}\right)$.

We assume that the energy dispersion of electrons in the beam is due to two terms. First, the dispersion of $R 1$ values across the pixel (based on Baptist et al. (1997) and Constancias (1998) (the dispersion of field enhancement factors across the pixel is assumed to be negligible in comparison). Second, the dispersion due to nanoprotrusions, described by an ad hoc parameter $\sigma_{n}$. The associated total dispersion can therefore be written:

$$
\sigma_{p}=\sigma_{p_{R}}+\sigma_{p_{n}}
$$

Differentiation of (5) relatively to $R 1$ allows to derive the resistive contribution:

$$
\delta V p_{R}=\frac{1}{A} \frac{\delta R 1}{R 1}\left[k i_{e}\left[R 1-\frac{B}{A}(1+\mu-A)\right]-V_{g c}\left(\frac{1+\mu}{A}-1\right)\right]
$$

The influence of nanometric structures can be in principle described by using the FowlerNordheim energy distribution, but is more complex to evaluate. A high field enhancement indeed occurs at the surface of the nanometric objects (Purcell et al., 1997), such that the electric field penetrates deeply into the material, allowing electrons from lower energy levels to 
be extracted. Therefore the corresponding energy spread is in principle proportional to the full width at half maximum of the Fowler-Nordheim distribution function that we recall here:

$$
\frac{d J}{d E} \cdot \frac{1}{J_{0}}=\frac{\exp \epsilon / d}{d\left[1+\exp \epsilon / k_{B} T\right]}
$$

where the Boltzmann constant is noted $k_{B}$, the temperature $\mathrm{T}, J_{0}$ is the emitted current density at zero temperature, $\epsilon=E-E_{F}$ the relative energy with respect to the Fermi level of the emitter, and d a function of the applied electric field $F\left(V . m^{-1}\right)$, and work function $\Phi(\mathrm{eV})$ of the emitter :

$$
d=0.93 .10^{-10} \frac{F}{\sqrt{\Phi}}
$$

Since the Full Width at Half Maximum of the FN distribution is related to parameter d (see Brenac et al. (1987) for instance) the $\sigma_{p_{n}}$ parameter derived from our measurements could in principle be used to estimate an equivalent electric field enhancement factor describing the influence of nanoprotrusions on the emission. Such calculations are however beyond the scope of the present study, but interesting to mention. Following the same approach than for deriving mean energy values, we calculate values of the parameter $\sigma_{p_{n}}$ by adjusting $\sigma_{p}$ to the energetic dispersion derived from the numerical simulation of the experiment, assuming a gaussian distribution of the energy of the emitted electrons.

We assume a value of $10 \%$ for $\frac{\delta R 1}{R 1}$ across the tips in one pixel. Table 1 shows adjusted values of parameters $R 1, V p_{n}$ and $\sigma_{p_{n}}$ obtained from the fit of measured currents as a function 
of Vgc.

\begin{tabular}{|l|l|l|l|l|l|l|l|}
\hline Vgc $(\mathrm{V})$ & 40 & 50 & 60 & 70 & 80 & 90 & 100 \\
\hline$R 1(k \Omega)$ & 340 & 340 & 340 & 340 & 340 & 340 & 340 \\
\hline$\frac{\delta R 1}{R 1}$ & 0.1 & 0.1 & 0.1 & 0.1 & 0.1 & 0.1 & 0.1 \\
\hline$V p_{R}(\mathrm{~V})$ & 0.76 & 1.41 & 2.41 & 3.97 & 6.19 & 9.33 & 13.49 \\
\hline$V p_{n}(\mathrm{~V})$ & 4.21 & 5.47 & 6.88 & 8.31 & 9.62 & 11.01 & 11.10 \\
\hline$\sigma_{p_{n}}(\mathrm{~V})$ & 0.65 & 1.04 & 1.31 & 1.65 & 1.86 & 1.92 & 1.97 \\
\hline$E_{\text {moy }}(\mathrm{eV})$ & 35.0 & 43.1 & 50.7 & 57.7 & 64.2 & 69.7 & 75.4 \\
\hline$\Delta E_{H W H M}(\mathrm{eV})$ & 1.15 & 1.73 & 2.25 & 2.89 & 3.45 & 3.97 & 4.45 \\
\hline
\end{tabular}

Tab. 1 -. Adjusted values of parameters describing the emission. Mean energy values and energy dispersion deduced from measurements are respectively noted $E_{\text {moy }}$ and $\Delta E_{H W H M}$.

Figure 16 shows the simulated mean energy and energy dispersion of electrons for each extraction voltage, constrained by our measurements. The energy dispersion increases with the emitted current, consistently with Purcell et al. (1997).

\subsection{Angular distribution of emitted electrons}

Null potentials are applied to all extraction grids except those located just above the emitting tips, therefore, the emitted beam is focalised along the $\mathrm{Y}$ axis, and defocalised along the $\mathrm{X}$ axis. This situation can be seen on Figures 10 and 11, which are respectively cuts along the $\mathrm{X}$ and $\mathrm{Y}$ axis, associated to the above configuration, for an idealized axisymetrical beam. The analysis of simulated electrons trajectories reveals that some electrons are deflected backward and will return to the cathode, most of the time charging the isolating layer (the grid to silica area ratio is about $15 \%$ ). 
Simulation results shown on Figures 10 and 11, have been obtained with the assumption of

a $45^{\circ}$ half angle dispersion of the beam. Over the set of five pixels tested we find an average half angle of $42^{\circ} \pm 20 \%$. We think that this value is representative of the actual angular dispersion of the tested sample. In our model the number of emitted electrons decreases as a cosine function of the emitted angle. This assumption is in good agreement with the observed profiles.

\section{Conclusions}

We used a hemispheric retarding field energy analyzer (Cipriani, 2006) to characterize the electron beam emitted by Mo microtips Field Emission Arrays made by the CEA/LETI laboratory. Those components integrate extraction grids and a resistive sheet. Measurements were carried out as a function of the extraction voltage, at normal incidence of the beam with respect to the analyzer entrance, without sample baking.

We derived the energy distribution of the electrons as a function of the extraction voltage with a resolution of $100 \mathrm{meV}$. Those measurements proved to be stable and repeatable. The mean energy and FWHM of the electrons are found to increase with the extraction voltage. The FWHM varies from $2 \mathrm{eV}$ at $40 \mathrm{~V}$ to $9 \mathrm{eV}$ at $100 \mathrm{~V}$, consistently with observations carried out in Purcell et al. (1997). As a consequence the mean energy is expected to vary across the emission life time of the device, at a rate depending both on the alteration of the resistive structure with time, and on the fate of adsorbed contaminants at the tip surface. We also found 
a mean angular dispersion of the beam of $42^{\circ} \pm 20 \%$, over the set of tested pixels. We used those observations to derive a simple phenomenological model of the emission accounting for the resistive structure of the component, as well as for the influence of nanoprotrusions on the emitted energy distribution, and for the mean angular dispersion of the beam. Such model can be used in order to design and optimize ion sources integrating future space instruments.

Space applications using FEA as electron guns suppose working in vacuum levels of the order of $10^{-6}$ mbar. The gaseous environment surrounding some planets and satellites in the Solar System, such as Mars or Europa, contains variable amounts of oxygen, carbon based molecules, and water vapor, which is also the main component outgassed from vessels structures. Some of those gases have been shown to be critical for the lifetime of FEA, including those based on microtips. For those reasons emission performances of such electron emitters, whatever their nature, have to be monitored in representative gaseous environments in order to infer their applicability to space applications.

\section{References}

Aplin, K. L., C. Collingwood, and B. Kent, 2004: Reliability tests of gated silicon field emitters for use in space. J. Phys. D.:Appl. Phys., 37, 2009-2017.

Baptist, R., L. Bachelet, and C. Constancias, 1997: Microtips and resistive sheet: A theoritical description of the emissive properties of the system. J. Vac. Sci. Techno. B, 15(2). 
Benenson, W., J. Harris, H. Stocker, and H. Lutz, 2002: Handbook of Physics. Springer.

Brenac, A., R. Baptist, G. Chauvet, and R. Meyer, 1987: Caractéristiques énergétiques de cathodes à micropointes à émission de champ. Revue Phys. Appl., 22, 1819-1834.

Cipriani, F., 2006: La couronne neutre suprathermique de Mars : modélisation et développement d'une instrumentation pour sa mesure in situ. Phd thesis, University of Versailles SaintQuentin-en-Yvelines.

Constancias, C., 1998: Emission d'Electrons à partir de Micropointes pour Ecrans Plats: Simulation, Caractérisation et Confrontations Experimentales. Thèse de doctorat, Université Joseph Fourier.

Purcell, S. T., Vu Thien Binh, and R. Baptist, 1997: Nanoprotrusion model for field emission from integrated microtips. J. Vac. Sci. Techno. B, 15(5), 1666-1677.

Schlesser, R., 1997: Energy distribution of field emitted electrons from diamond coated molybdenum tips. Appl. Phys. Lett., 70(12), 33-35.

Spindt, C. A., I. Brodie, L. Humphrey, and E. R. Westerberg, 1976: Physical properties of thin-film field emission cathodes with molybdenum cones. J. Appl. Phys., 47(12), 52485263.

Temple, D., 1999: Recent progress in field emitter array developpment for high performance applications. Mat. Sicence and Eng. 185-239. 


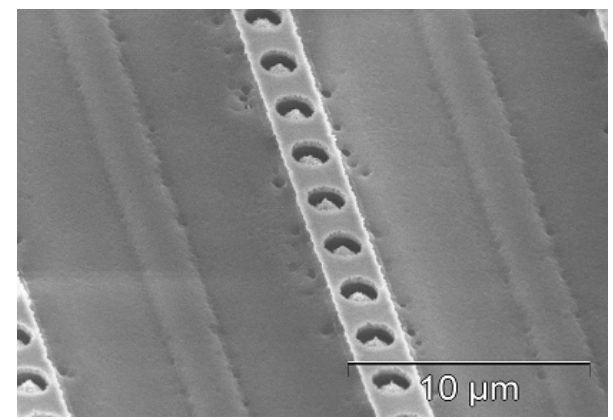

Fig. 1 -. SEM image showing extraction grid holes $(\phi=1.4 \mu m)$, and underlying Mo tips. The mean distance between two holes centers is $3 \mu \mathrm{m}$.

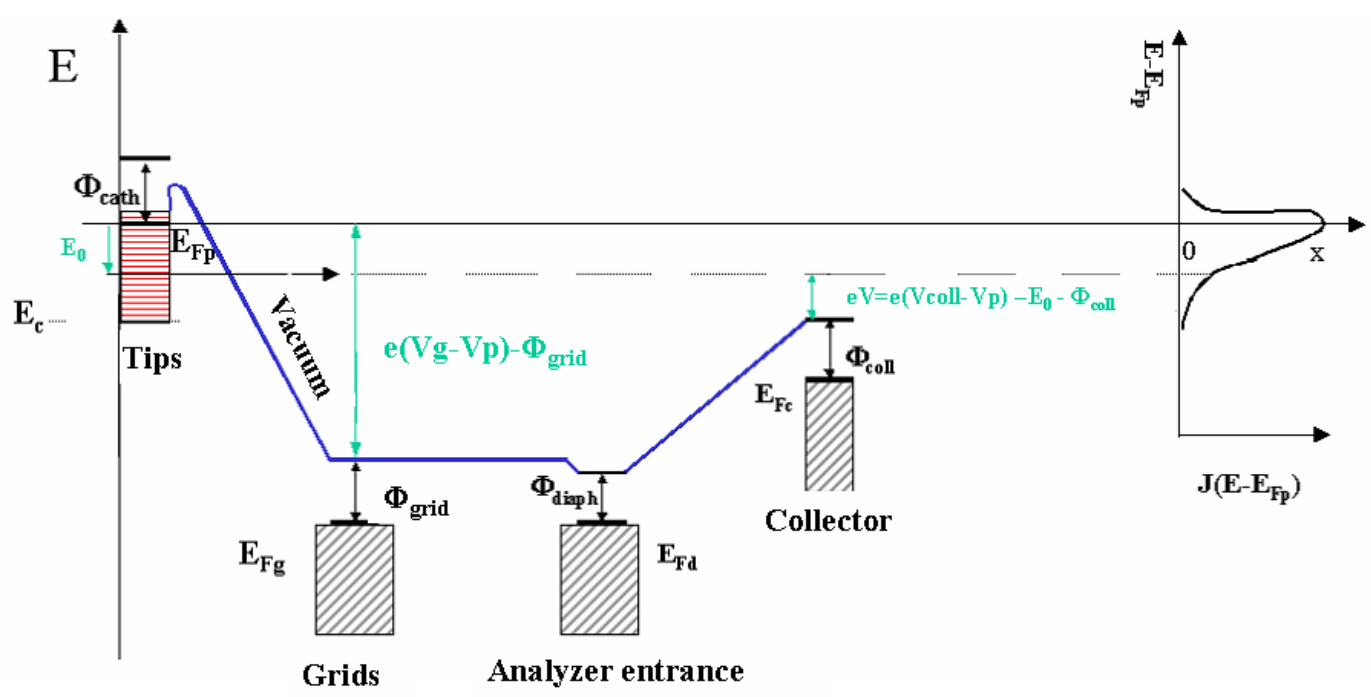

Fig. 2 -. Potential energy diagram of the experiment. Electrons having an energy $E_{0}$ with respect to the Fermi level of the tips material leave the tips at potential Vp, and are accelerated towards the grids at potential $V$ g. They have an energy close to the grids equal to $e(V g-V p)-\Phi_{\text {grid }}$ where $\Phi_{\text {grid }}$ is the work function of the grid material. Electrons leaving the grids are further accelerated towards the entry plane of the analyzer at potential Vd. They enter the retarding field controlled by (Vcoll- $V d)$. Their energy close to the collector surface is $e(V$ coll $-V p)-E_{0}-\Phi_{\text {coll }}$, where $\Phi_{\text {coll }}$ is the collector's work function. 

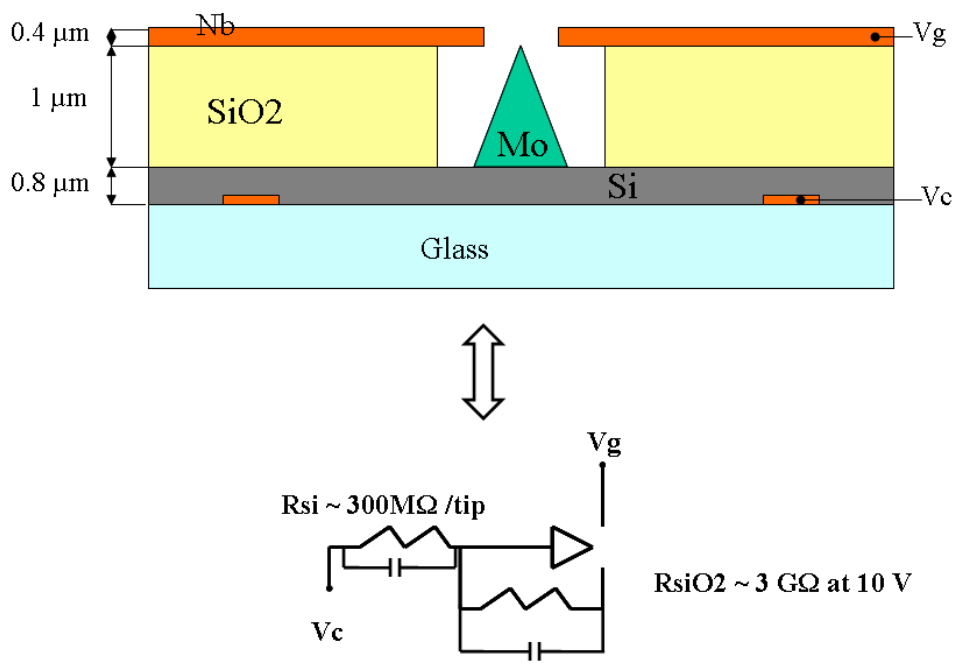

Fig. 3 -. Emitting unit structure and dimensions. A molybdenum tip is represented (in green), two cathodic contacts and extraction grids made of Niobium (orange), the silica insulating layer (yellow), and the resistive sheet made of silicion (grey). The equivalent electrical structure per tip is also shown. 


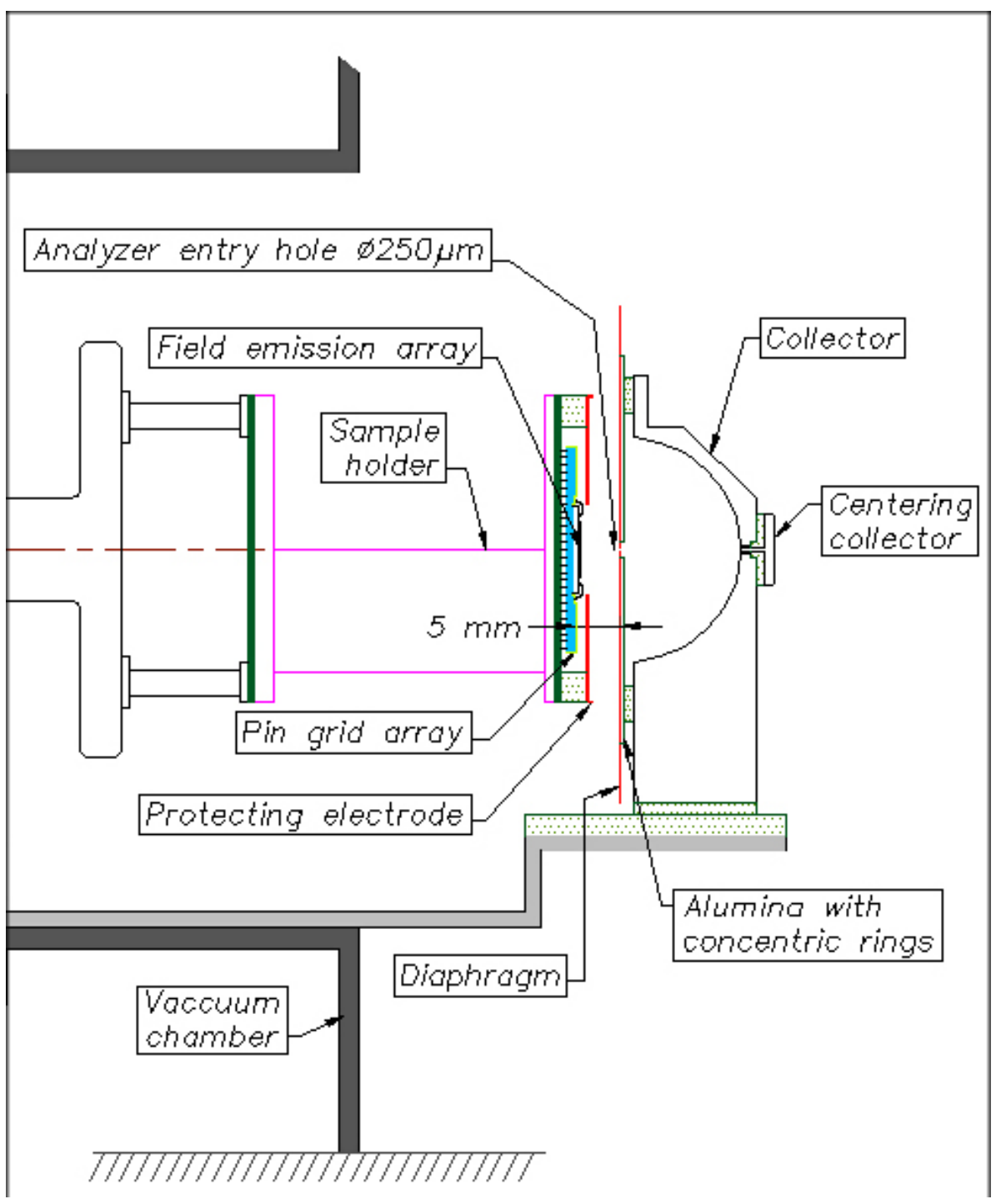

Fig. 4 -. Experimental setup overview. The FEA is bonded in a PGA cavity (blue part on the drawing), plugged into an alumina support (green part). The emitting surface is parallel to the entrance plane of the analyzer (red line, named Diaphragm), which has a small entry hole (diameter $250 \mu \mathrm{m})$. An alumina support with conducting concentric rings is placed at the back of the entrance plane, in front of the hemispheric collector. The on-axis part of the collector (centering collector) is a $1 \mathrm{~mm}^{2}$ area insulated from the main collector part, allowing centering of the emitter with respect to the analyzer entry hole. 


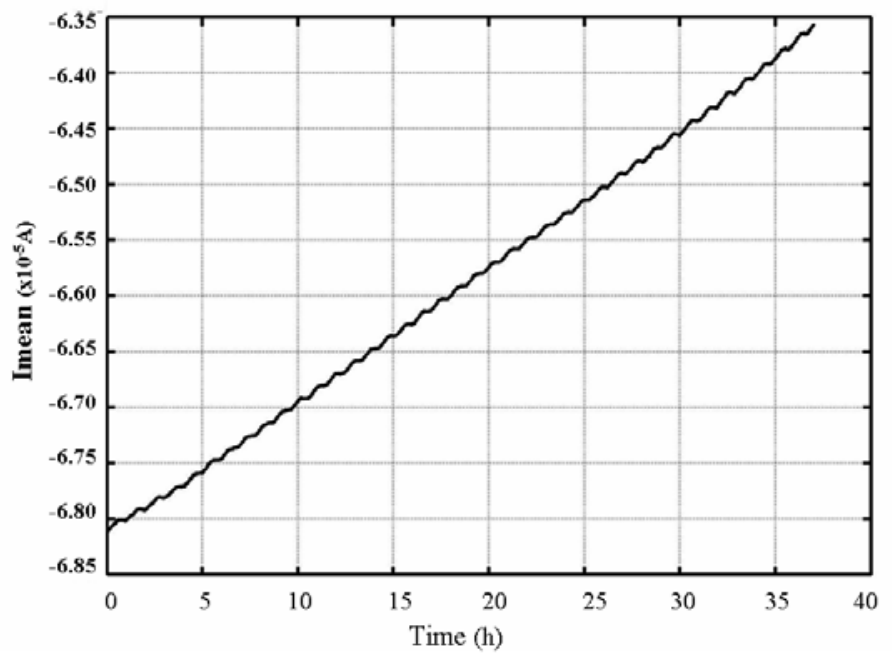

Fig. 5 -. Variations over 30 h of the emitted current (one column of 32 pixels), for an extraction potential of $90 \mathrm{~V}$.

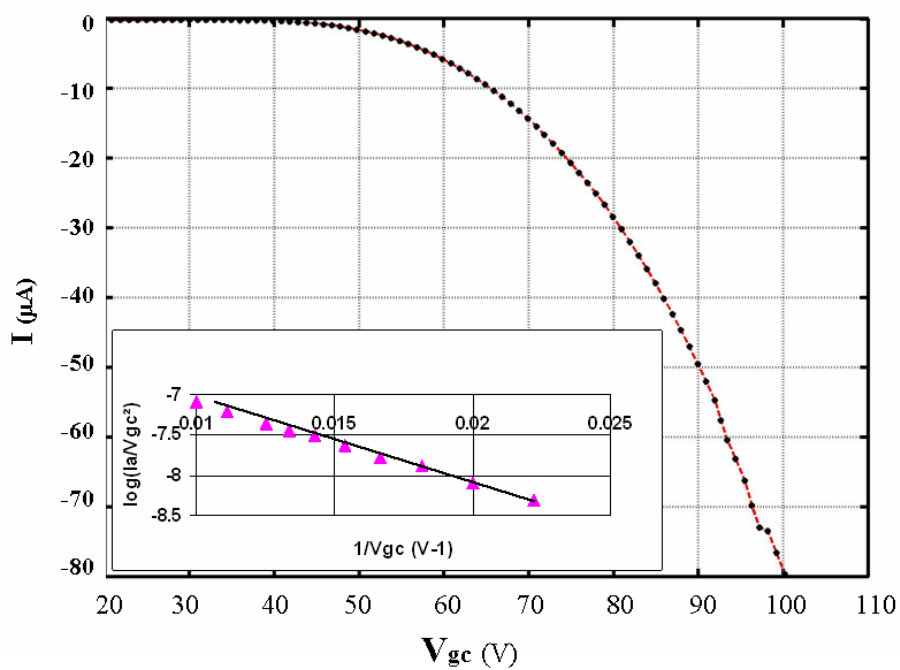

Fig. 6 -. Typical courant-voltage plot for one stabilized 32 pixels column. The inset shows the associated Fowler-Nordheim plot. 


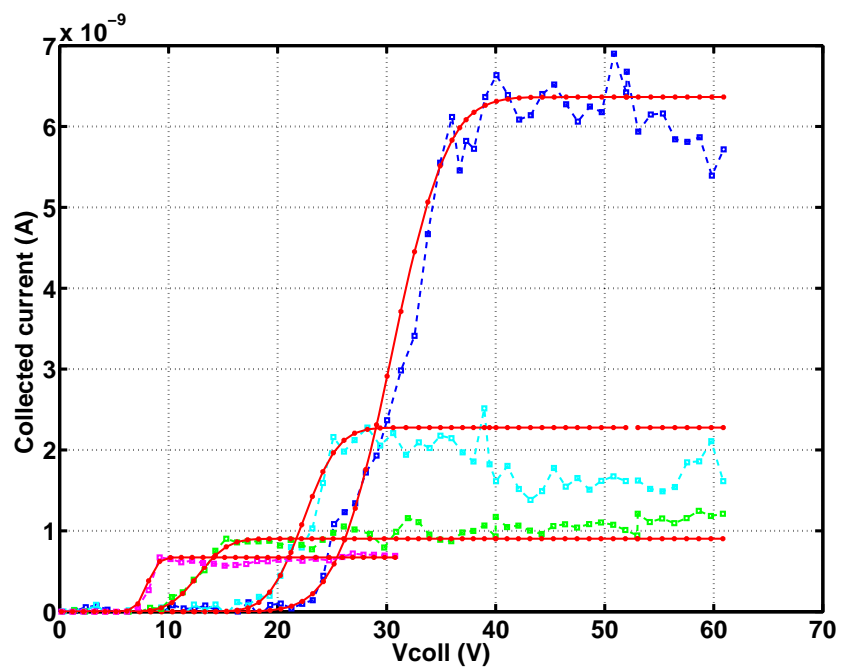

Fig. 7 -. Comparison of collector currents when Pixel $(17,15)$ is emitting with an extraction voltage successively set to $40 \mathrm{~V}$ (magenta curve), 60V(green curve), 80V(cyan curve) and 100 V(blue curve). Measurements are fitted (red curves) with a simulation of the experiment based on the emitter model presented in part 5.

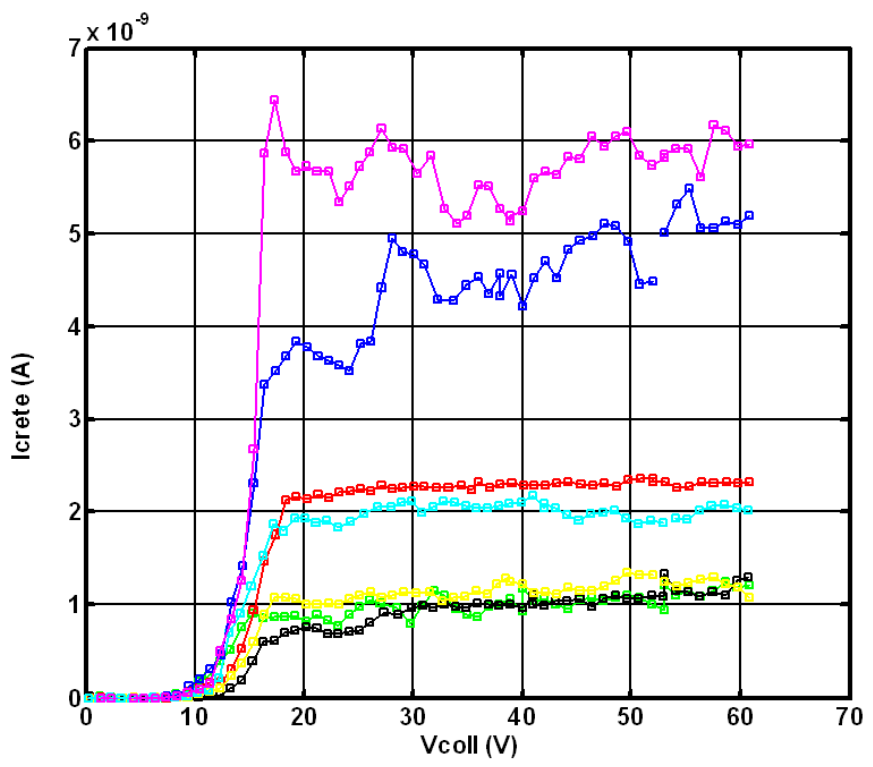

Fig. 8 -. Comparison of collected currents at an extraction voltage of $60 \mathrm{~V}$ for different pixels:(17,15) 1-green, (17,15) 2-black, (9,14) 1-red, (9,14) 2-yellow, (27,8) cyan, (9,16) blue, $(9,15)$ magenta. 


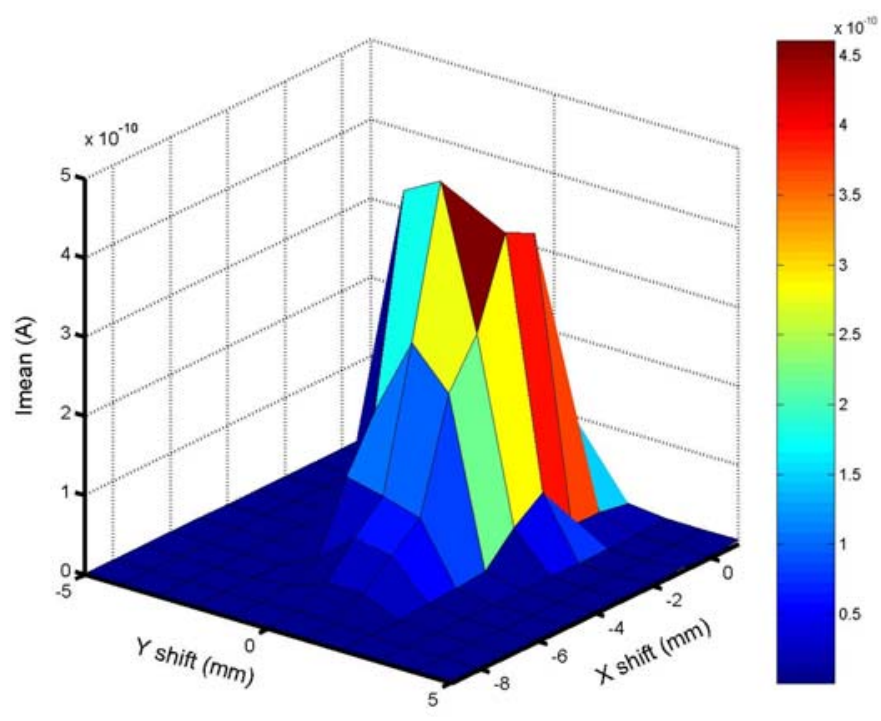

Fig. 9 -. 3D profil of the collected current for pixel(9,15), whith $V g c=60 V, V d=80 \mathrm{~V}$. The current reported on the $Z$ axis is a mean value (duty cycle of 10\%).

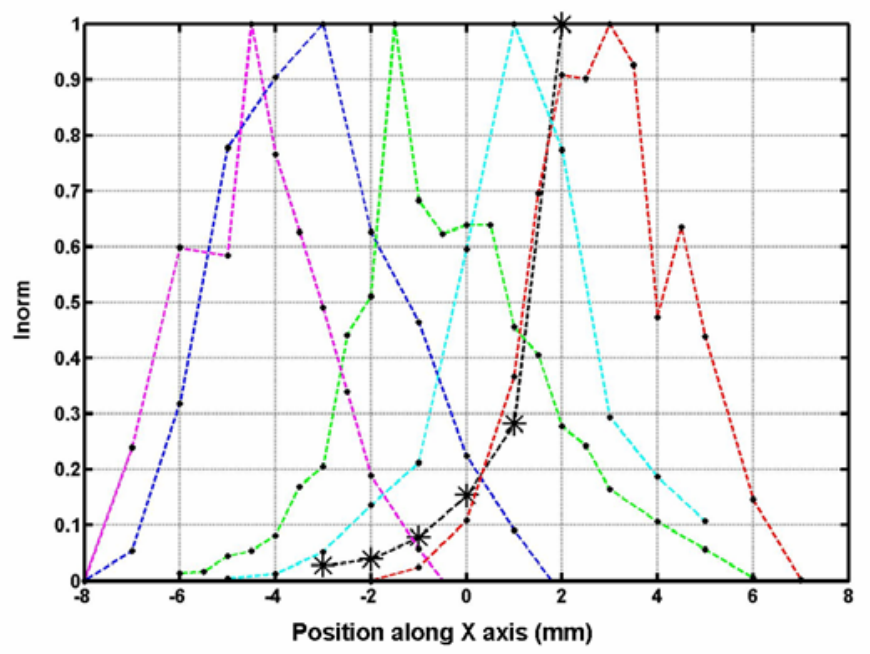

Fig. 10 -. Normalized collected current as a function of the emitter position along the $X$ axis, for pixel:(17,15) green, $(9,14)$ blue, $(27,8)$ red, $(9,16)$ magenta, $(9,15)$ cyan. 


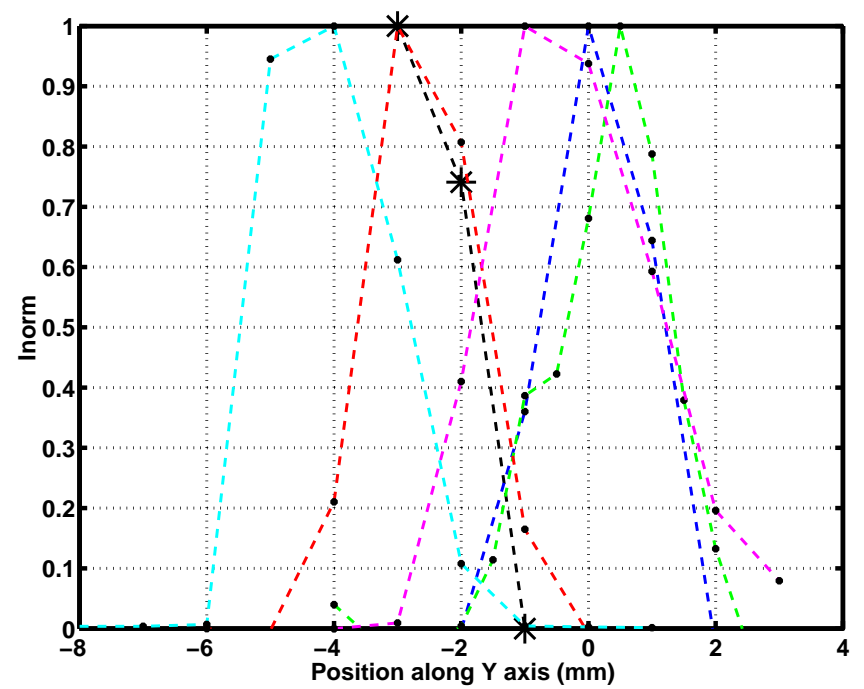

Fig. 11 -. Normalized collected current as a function of the emitter position along the $Y$ axis, for pixel:(17,15) green, $(9,14)$ blue, $(27,8)$ red, $(9,16)$ magenta, $(9,15)$ cyan.

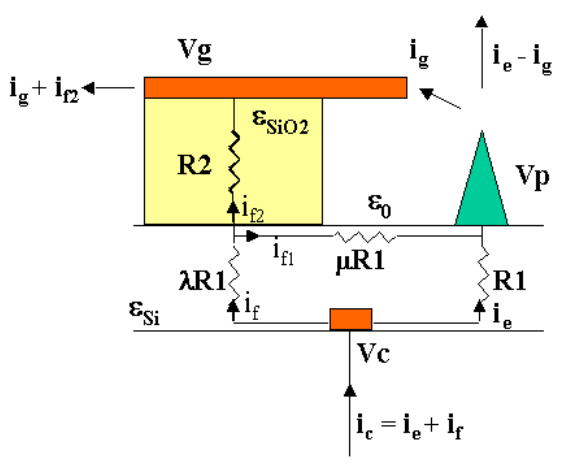

Fig. 12 -. Resistive structure of the emitting unit (a detailed description is given in the text). 


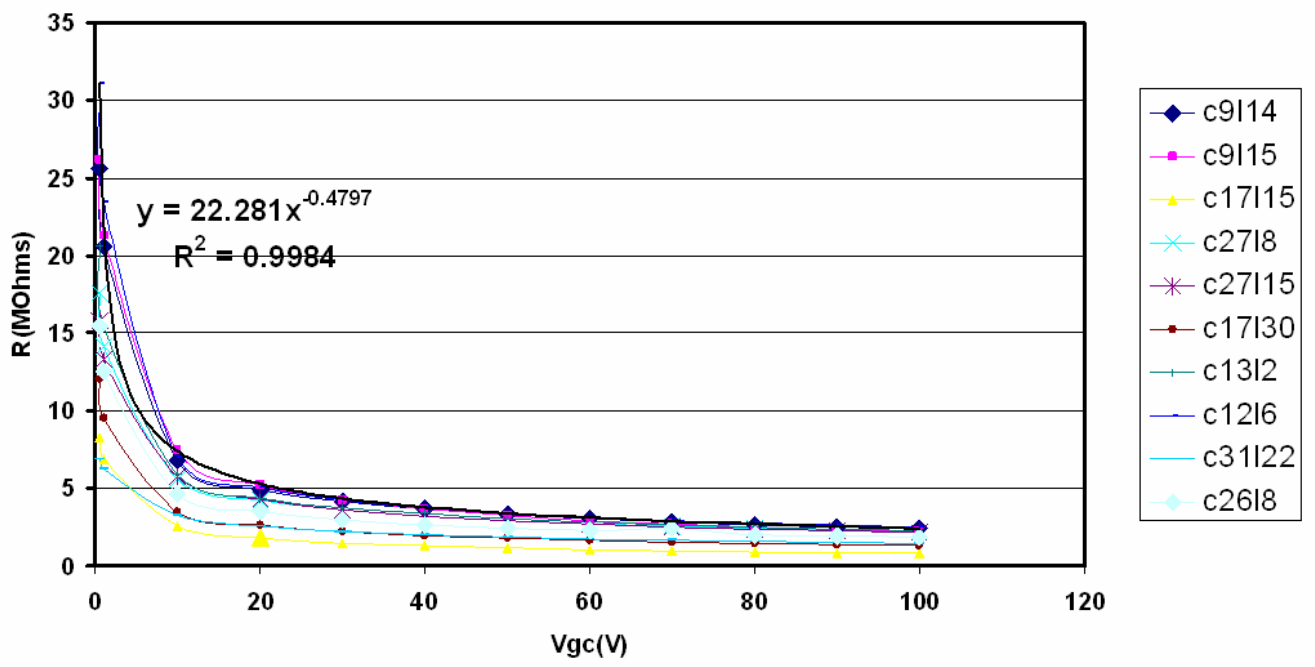

Fig. 13 -. Evolution of the isolation between the grids and the cathodic contacts as a function of an inverse extraction voltage. 


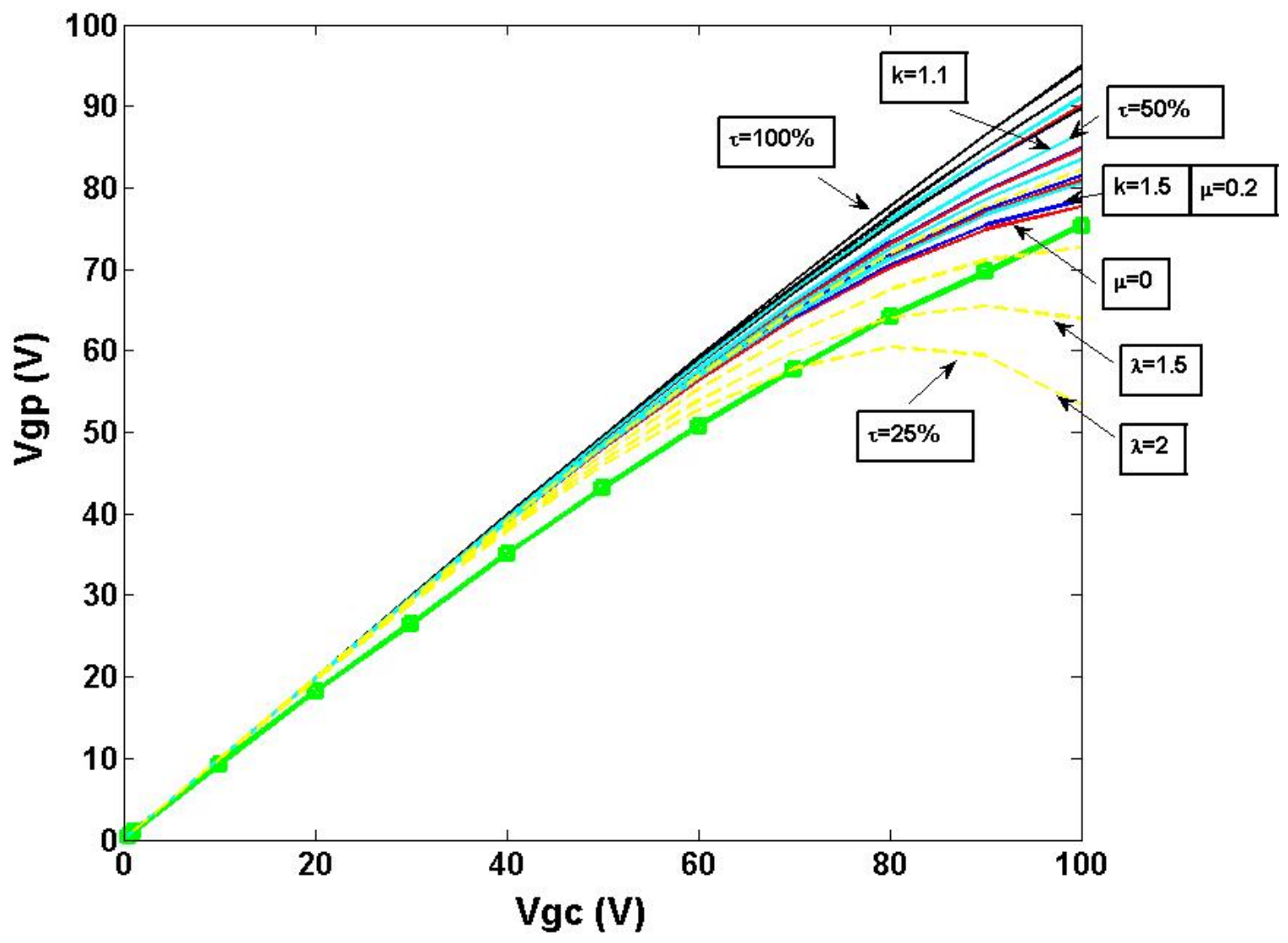

Fig. 14 -. Potential difference between the extraction grid and tips (Vg-Vp) noted Vgp, as a function of the applied voltage Vgc. The green curve represents values of Vgp deduced from measurements of Figure 12. Details on curve families are given in the text. 


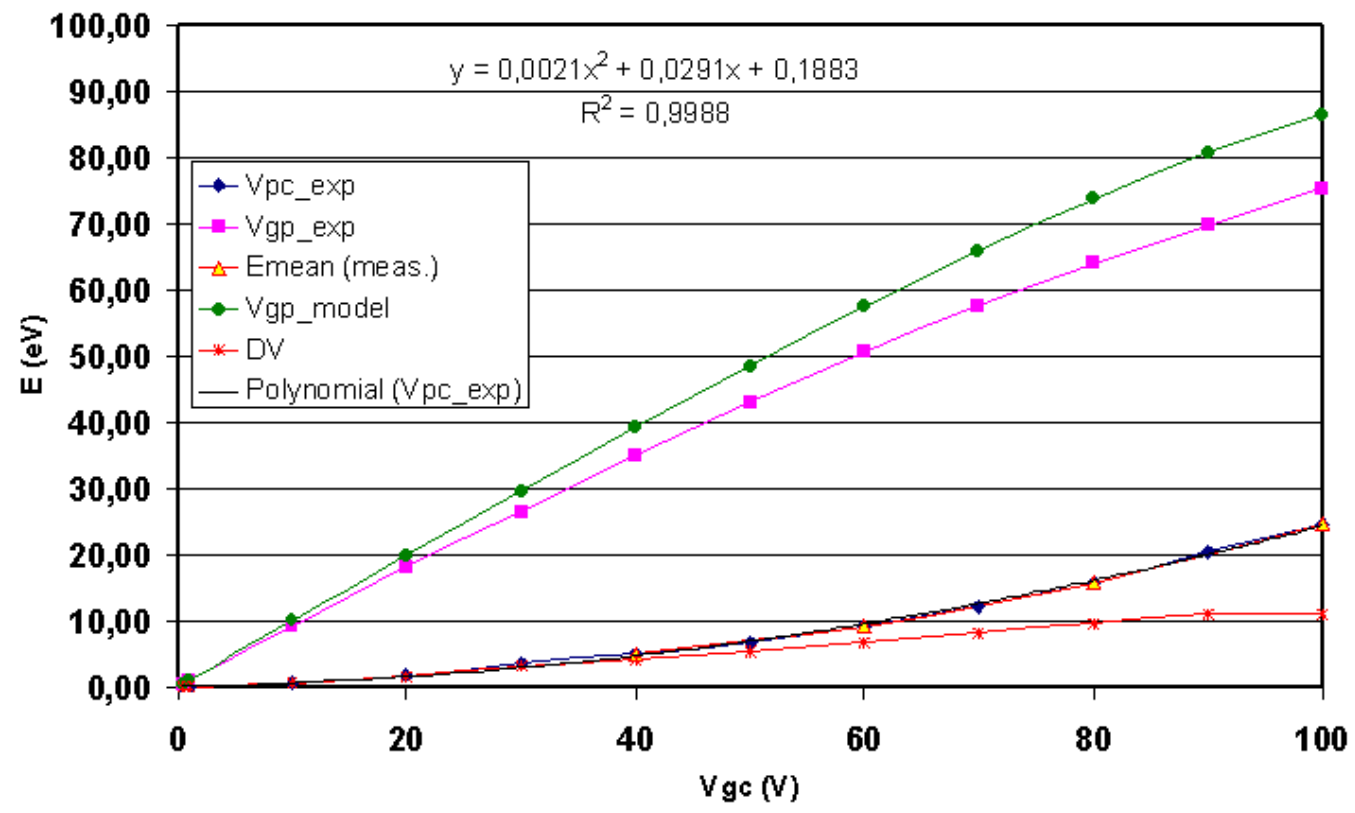

Fig. 15 -. Comparison of the modelled and measured energy shift as a function of the extraction voltage Vgc. 

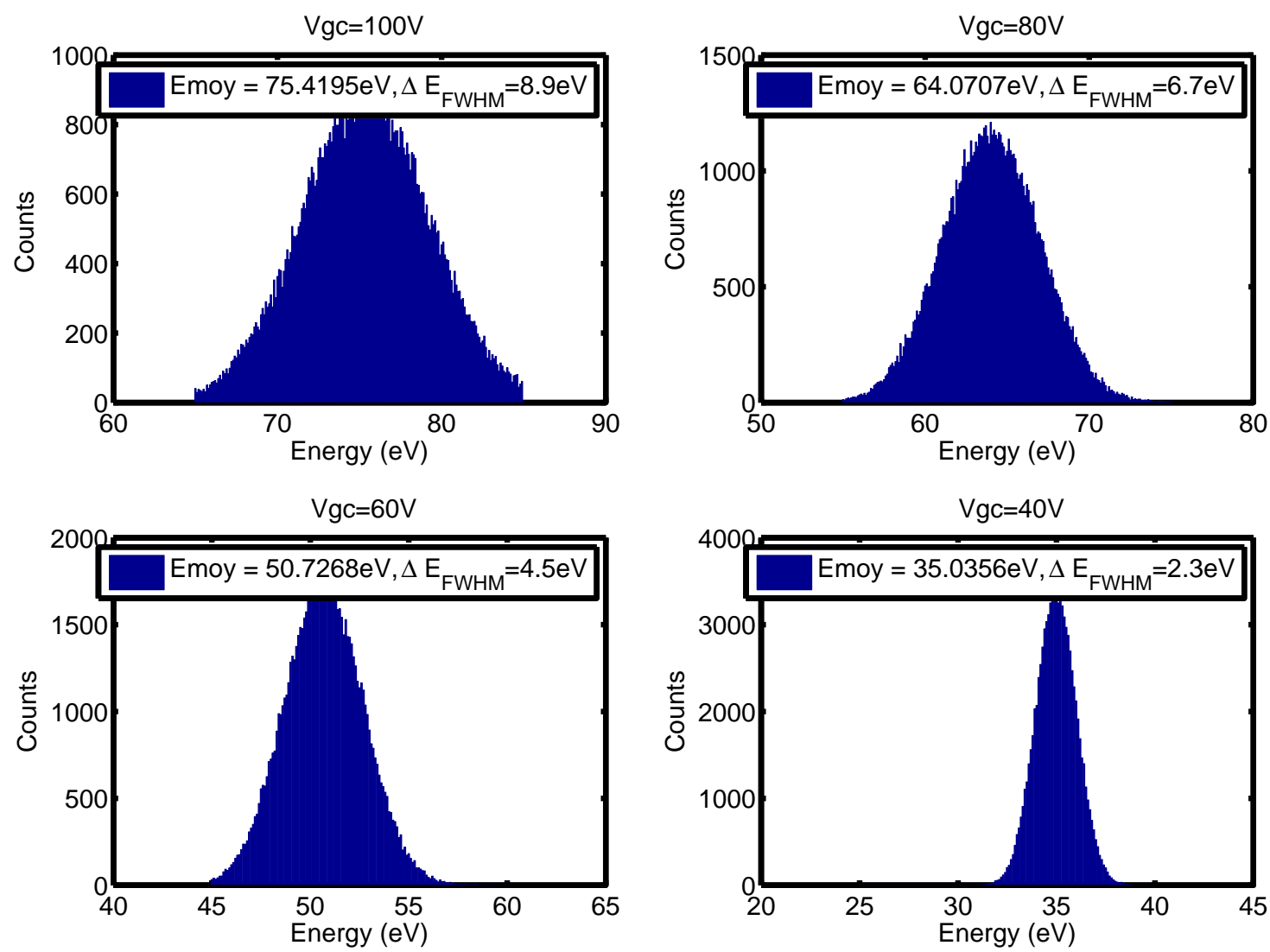

Fig. 16 -. Simulated energy distributions for each extraction voltage. 